\title{
FITOSSOCIOLOGIA DE CERRADO Sensu Stricto NO MUNICÍPIO DE ABAETÉ-MG ${ }^{1}$
}

\author{
Amilcar Walter Saporetti Jr. ${ }^{2}$, João Augusto Alves Meira Neto 3 , e Roosevelt de Paula Almado ${ }^{4}$
}

\begin{abstract}
RESUMO - O cerrado tem sido objeto de discussão de grupos temáticos que estudam a conservação de biodiversidade no Estado de Minas Gerais. São inúmeras as áreas de conservação com vegetação de cerrado das quais não se têm informações a respeito de sua composição florística e estrutura. O objetivo deste trabalho foi avaliar florística e fitossociologicamente uma área de cerrado sensu stricto, no município de Abaeté-MG. A área de estudo é um fragmento com 2 ha de cerrado sensu stricto, preservado como área de reserva da CAF-Santa Bárbara, situada nas coordenadas $19^{\circ} 05^{\prime} \mathrm{S}$ e $44^{\circ} 58^{\prime} \mathrm{W}$, a uma altitude de $480 \mathrm{~m}$, em leve depressão próxima de uma vereda. O clima pertence ao tipo Cwa pelo sistema de Köppen, com precipitação média anual de $1.400 \mathrm{~mm}$. O solo é do tipo Latossolo Vermelho distrófico. Foram instaladas 15 parcelas de $200 \mathrm{~m}^{2}(10 \times 20 \mathrm{~m})$, distribuídas sistematicamente ao longo de trilhas, distanciadas $10 \mathrm{~m}$ entre si. Foram amostrados todos os indivíduos lenhosos vivos com circunferência do caule ao nível do solo (CAS) igual ou maior que $10 \mathrm{~cm}$. O índice de Shannon foi de 3,590 e a equabilidade foi de 0,804 , considerados comuns para cerrados bem conservados. Foram amostrados 1.339 indivíduos, sendo a composição florística constituída por 85 espécies, distribuídas em 44 famílias. As famílias com maior número de espécies foram Leguminosae Caesalpinioideae com sete espécies, Annonaceae com cinco, Myrtaceae, Malpighiaceae, Erythroxylaceae, Anacardiaceae, Rubiaceae e Bignoniaceae com quatro, seguidas de Vochysiaceae e Leguminosae Papilionoideae com três. As espécies que apresentaram o maior valor de importância (VI) foram Xylopia aromatica (Lam.) Mart. (22,21), Myrcia lingua Berg $(18,18)$ Caryocar brasiliense Cambess. (17,91), Eugenia dysenterica DC. $(17,58)$, Byrsonima intermedia A. Juss. $(13,69)$ e Brosimum gaudichaudii Trécul $(11,86)$.
\end{abstract}

Palavras-chave: Composição florística, estrutura, conservação.

\section{PHYTOSOCIOLOGY OF THE CERRADO SENSU STRICTO IN ABAETÉ, MG, BRAZIL}

\begin{abstract}
The cerrado has been a topic of discussion of thematic groups studying biodiversity conservation in the state of Minas Gerais, Brazil. No information is available on floristic composition and structure of innumerous conservation areas with a cerrado vegetation. The objective of this work was to perform a floristic and phytosociologic survey of a cerrado sensu stricto area in the municipality of Abaete, as a floristic-structural reference of the cerrados in the region. The studied area is a fragment with 2 ha of cerrado sensu stricto, preserved as a CAF - Santa Bárbara reserve area. It is located within the coordinates $19^{\circ} 05^{\prime} \mathrm{S}$ and $44^{\circ} 58^{\prime} \mathrm{W}$, in a smooth depression close to a riparian area. The type of soil is Distrophic Red Latosol. The latitude is $480 \mathrm{~m}$. The climate is of the Cwa type by the Koeppen system and the annual mean precipitation is $1.400 \mathrm{~mm}$. A total of 15 plots of $200 \mathrm{~m}^{2}(10 \times 20 \mathrm{~m})$ sistematically distributed along $10 \mathrm{~m}$ spacing paths weree stablished. The criterium of inclusion was a stem circumference of $10 \mathrm{~cm}$ at ground level (CGL), sampling all alive individuals. The Shannon Index was 3.590, and the equability of 0.804 , considered to be common values for well-preserved cerrados. A total of 1.339 individuals were sampled with the floristic composition of Abaeté being constituted by 85 species, distributed within 44 families. The families with greater number of species are Leguminosae Caesalpinioideae with 7 species, Annonaceae with 5, Myrtaceae, Malpighiaceae, Erythroxylaceae, Anacardiaceae, Rubiaceae and Bignoniaceae with 4, and Vochysiaceae, Leguminosae Papilionoideae with 3 species. The species showing greater IV were Xylopia aromatica (Lam.) Mart. (22,21), Myrcia lingua Berg (18,18) Caryocar brasiliense Cambess. (17,91), Eugenia dysenterica DC. (17,58), Byrsonima intermedia A. Juss. (13,69) and Brosimum gaudichaudii Trécul $(11,86)$.
\end{abstract}

Key words: $\quad$ Floristic composition, structure, conservation.

1 Recebido para publicação em 7.8.2002.

Aceito para publicação em 12.5.2003.

2 Graduado em Ciências Biológicas, Dep. de Biologia Vegetal da Universidade Federal de Viçosa - UFV, <amilcarjr@iname.com>; ${ }^{3}$ Prof. Dr. do Dep. de Biologia Vegetal da UFV, <j.meira@ mail.ufv.br>; ${ }^{4}$ CAF - Santa Bárbara Ltda. 
O cerrado tem sido objeto de discussão de grupos temáticos que estudam a conservação de biodiversidade no Estado de Minas Gerais. Na distribuição do cerrado no Estado, foi definida uma Área de Alta Importância Biológica denominada Bom Despacho, que engloba os municípios de Bom Despacho, Martinho Campos e Dores do Indaiá. Para essa área, foram estabelecidas recomendações para conservação da biodiversidade. Essas recomendações são, pela ordem, criação de unidades de conservação, investigação científica e recuperação de áreas degradadas (FUNDAÇÃO BIODIVERSITAS, 1998). O município de Abaeté, local da área de estudos deste trabalho, é vizinho do município de Martinho Campos.

Há necessidade de informações florístico-estruturais dos cerrados da região. A natureza fragmentar de áreas remanescentes de cerrado implica uma série de procedimentos que deveriam ser praticados para reverter o processo de deterioração das reservas (Meira-Neto, 1991).

O objetivo deste trabalho foi avaliar florística e fitossociologicamente um cerrado sensu stricto para servir de referencial para a escolha de áreas para futuras unidades de conservação e para projetos de recuperação de áreas degradadas dos cerrados da região.

A área de estudos é um fragmento de Cerrado sensu stricto com 2 ha, pertencentes à Companhia Agrícola Florestal - Santa Bárbara Ltda. (CAF - Santa Bárbara Ltda.), preservado como área de reserva da empresa, situado nas coordenadas $19^{\circ} 05^{\prime} \mathrm{S}$ e $44^{\circ} 58^{\prime} \mathrm{W}$, a uma altitude de $480 \mathrm{~m}$. O solo é do tipo Latossolo Vermelho distrófico. A precipitação média anual é de $1.400 \mathrm{~mm}$ e o clima pertence ao tipo Cwa pelo sistema de Köppen.

O método fitossociológico aplicado foi o de parcelas (Mueller-Dombois \& Ellenberg, 1974), tendo sido efetuada uma amostra que perfazia 0,3 ha de área amostral.

Foi elaborada uma lista florística das espécies amostradas. A identificação taxonômica das espécies avaliadas foi efetuada mediante consultas a herbários, consultas a especialistas e por meio de literatura especializada. $\mathrm{O}$ sistema de classificação utilizado foi o de Cronquist (1988).

Para o estudo foram instaladas 15 parcelas de $200 \mathrm{~m}^{2}$ $(10 \times 20 \mathrm{~m})$ na área amostral. A distribuição das parcelas foi feita sistematicamente ao longo de trilhas, distanciadas

R. Árvore, Viçosa-MG, v.27, n.3, p.413-419, 2003
$10 \mathrm{~m}$ entre si. Foram amostradas todas as plantas lenhosas eretas com circunferência do caule à altura do solo (CAS) maior ou igual a $10 \mathrm{~cm}$. Os parâmetros fitossociológicos calculados foram densidade, frequiência e dominância absolutas e relativas e, a partir destas, o valor de importância (Mueller-Dombois \& Ellenberg, 1974). Foram calculados o índice de diversidade de Shannon (H') e a equabilidade (J') para a amostra (PIELOU, 1975) como indicadores de heterogeneidade. Para calcular os referidos parâmetros foi utilizado o software Fitopac 1 (Shepherd, 2001).

Foram relacionadas 85 espécies de 44 famílias botânicas. As famílias com maior número de espécies foram Leguminosae Caesalpinioideae com sete espécies, Annonaceae com cinco, Myrtaceae, Malpighiaceae, Erythroxylaceae, Anacardiaceae, Rubiaceae e Bignoniaceae com quatro, seguidas de Vochysiaceae, Leguminosae Papilionoideae com três (Quadro 1).

Foram amostrados 1.339 indivíduos em 0,3 ha, correspondendo a uma densidade total (DT) de 4.463,33 indivíduos por hectare. A altura máxima das espécies lenhosas foi de $14,5 \mathrm{~m}$, a altura média foi de $3,56 \mathrm{~m}$ e a altura mínima foi de $0,30 \mathrm{~m}$. O diâmetro médio das plantas amostradas foi de 7,87 $\mathrm{cm}$. Para as espécies o índice de diversidade de Shannon (H') foi de 3,590 e a equabilidade (J') 0,804. Para uma comparação direta dos índices, sem maiores preocupações com a significância, é possível relacionar cerrados bem conservados, em unidades de conservação, com índices aproximados (Quadro 2).

A espécie que apresentou a maior densidade relativa (DR) foi Xylopia aromatica com 9,63\%, seguida de Myrcia lingua (8,74\%), Eugenia dysenterica e Byrsonima intermedia com 6,72\%, Brosimum gaudichaudii (5,75\%), Erythroxylum daphnites (3,88\%) e Byrsonima verbascifolia $(3,81 \%)$. As espécies que apresentaram maior dominância relativa (DoR) foram Caryocar brasiliense (12,27\%), Xylopia aromatica $(9,18 \%)$, Eugenia dysenterica (7,91\%), Myrcia lingua $(6,04 \%)$, Qualea grandiflora e Astronium fraxinifolium (4,43\%), e Erythroxylum daphnites (3,98\%). As espécies que apresentaram o maior valor de importância (VI) foram Xylopia aromatica $(22,21)$, Myrcia lingua $(18,18)$, Caryocar brasiliense (17,91), Eugenia dysenterica $(17,58)$, Byrsonima intermedia $(13,69)$ e Brosimum gaudichaudii $(11,86)$ (Quadro 3). 
Quadro 1 - Relação de espécies lenhosas amostradas no cerrado da CAF, município de Abaeté-MG

Table 1 - Relation of woody species sampled in the CAF's cerrado vegetation, in Abaeté-MG, Brazil

\begin{tabular}{|c|c|c|}
\hline Família & Espécie & Nome Vulgar \\
\hline \multirow{4}{*}{ 1-Anacardiaceae } & Astronium fraxinifolium Schott & Gonçalves \\
\hline & Lithraea molleoides (Vell.) Engl. & Aroeirinha \\
\hline & Myracrodruon urundeuva Allemão & Aroeira-verdadeira \\
\hline & Tapirira guianensis Aubl. & Pau-pombo \\
\hline \multirow{5}{*}{ 2-Annonaceae } & Annona coriacea Mart. & Marolo \\
\hline & Annona crassiflora Mart. & Araticum/marolo \\
\hline & Annona dioica A.St.-Hil. & Araticum/marolo \\
\hline & Duguetia furfuracea (A.St.-Hil.) Benth. \& Hook. & Araticum \\
\hline & Xylopia aromatica (Lam.) Mart. & Pindaíba \\
\hline 3-Apocynaceae & Aspidosperma tomentosum Mart. & Tambú \\
\hline 4-Araliaceae & Didymopanax macrocarpum Seem. & Mandioqueira \\
\hline 5-Arecaceae & Syagrus flexuosa (Mart.) Becc. & \\
\hline \multirow{2}{*}{ 6-Asteraceae } & Piptocarpha rotundifolia (Less.) Baker & Macieira \\
\hline & Vernonia polyanthes (Spreng.) Less. & Assapeixe-branco \\
\hline \multirow{4}{*}{ 7-Bignoniaceae } & Cybistax antisyphilitica (Mart.) Mart. & Ipê de flor verde \\
\hline & Tabebuia caraiba (Mart.) Bureau & Caraíba \\
\hline & Tabebuia ochracea (Cham.) Standl.' & Ipê \\
\hline & Zeyheria digitalis (Vell.) Hoehne \& Kuhlm. & Saco-de-bode/bolsa-de-pastor \\
\hline 8-Burseraceae & Protium heptaphyllum (Aubl.) March. & Amescla \\
\hline \multirow{7}{*}{ 9-Leguminosae Caesalpinioideae } & Acosmium dasycarpum (Vogel) Yakovlev & Chapada \\
\hline & Bauhinia bongardii Steud. & Pata-de-vaca/unha-de-bezerro \\
\hline & Bauhinia holophylla Steud. & Pata-de-vaca/unha-de-bezerro \\
\hline & Dimorphandra mollis Benth. & Faveiro \\
\hline & Hymenaea stigonocarpa Mart. & Jatobá \\
\hline & Sclerolobium paniculatum Vogel & Angá \\
\hline & Senna rugosa (G. Don) Irwin \& Barneby & Canafístula \\
\hline 10-Caryocaraceae & Caryocar brasiliense Cambess. & Pequi \\
\hline 11-Cecropiaceae & Cecropia pachystachya Trécul & Embaúba \\
\hline \multirow{2}{*}{ 12-Clusiaceae } & Kielmeyera coriacea (Spreng.) Mart. & Pau-santo \\
\hline & Kielmeyera variabilis Mart. & Gordinho \\
\hline 13-Combretaceae & Terminalia argentea Mart. & Capitão-do-cerrado \\
\hline \multirow{2}{*}{ 14-Connaraceae } & Connarus suberosus Planch. & \\
\hline & Rourea induta Planch. & \\
\hline 15-Chrysobalanaceae & Couepia grandiflora Benth. & Fruto de ema, emeira \\
\hline \multirow{2}{*}{ 16-Dilleniaceae } & Curatella americana $\mathrm{L}$. & Pau-de-lixa \\
\hline & Davilla rugosa Poir. & Lixeirinha \\
\hline 17-Ebenaceae & Diospyros hispida A. DC. & Caqui-do-cerrado \\
\hline \multirow{4}{*}{ 18-Erythroxylaceae } & Erythroxylum daphnites Mart. & Zezinho \\
\hline & Erythroxylum suberosum A.St.-Hil.' & Mercúrio-do-campo \\
\hline & Erythroxylum sp. & \\
\hline & Erythroxylum tortuosum Mart. & Mercúrio-do-campo \\
\hline
\end{tabular}


Quadro 1, cont.

Table 1, cont.

\begin{tabular}{|c|c|c|}
\hline Família & Espécie & Nome Vulgar \\
\hline \multirow{3}{*}{ 19-Leguminosae Papilionoideae } & Andira fraxinifolia Benth. & Angelim \\
\hline & Bowdichia virgilioides H.B.K. & Sucupira-roxa \\
\hline & Machaerium opacum Vogel & Jacarandá-roxo \\
\hline \multirow{2}{*}{ 20-Flacourtiaceae } & Casearia gossypiosperma Briq. & Pau-de-lagarto/erva-de-lagarto \\
\hline & Casearia sylvestris Sw. & Pau-de-lagarto/erva-de-lagarto \\
\hline 21-Lamiaceae & Hyptis cana Pohl ex Benth. & Candeia \\
\hline 22-Loganiaceae & Strychnos pseudoquina A. St.-Hil. & Quina-do-cerrado \\
\hline 23-Lythraceae & Lafoensia pacari A.St.-Hil. & Pacari, dedaleiro \\
\hline \multirow{4}{*}{ 24-Malpighiaceae } & Banisteriopsis anisandra (A. Juss.) B. Gates & \\
\hline & Byrsonima coccolobifolia $\mathrm{H}$. B. K. & Cajuzinho \\
\hline & Byrsonima intermedia A. Juss. & Canela/muricizinho \\
\hline & Byrsonima verbascifolia (L.) Rich. ex A. Juss. & Muricizão \\
\hline \multirow{2}{*}{ 25-Melastomataceae } & Miconia albicans (Sw.) Triana & $\mathrm{Cu}$-de-pinto \\
\hline & Miconia rubiginosa (Bonpl.) DC. & Quaresminha \\
\hline 26-Meliaceae & Cabralea canjerana (Vell.) Mart. & Canjerana \\
\hline \multirow{2}{*}{ 27- Leguminosae Mimosoideae } & Enterolobium gummiferum (Mart.) J. F. Macbr. & Vinhático-abóbora \\
\hline & Stryphnodendron adstringens (Mart.) Coville & Barbatimão \\
\hline 28-Monimiaceae & Siparuna guianensis Aubl. & Catinga-d'anta \\
\hline 29-Moraceae & Brosimum gaudichaudii Trécul & Mama-cadela \\
\hline \multirow{2}{*}{ 30-Myrsinaceae } & Cybianthus detergens Mart. & Mangabinha \\
\hline & Rapanea umbellata (Mart.) Mez & Capororoca \\
\hline 31-Myristicaceae & Virola sebifera Aubl. & Café-do-mato \\
\hline \multirow{4}{*}{ 32-Myrtaceae } & Eugenia dysenterica DC. & Cagaita \\
\hline & Myrcia lingua Berg & Goiabinha \\
\hline & Myrcia rostrata $\mathrm{DC}$. & Folha-miúda \\
\hline & Myrcia tomentosa (Aubl.) DC. & Goiabeira-do-mato \\
\hline 33-Ochnaceae & Ouratea spectabilis (Mart.) Engl. & Gordinha \\
\hline 34-Opiliaceae & Agonandra brasiliensis Benth. \& Hook. f. & Carobinho \\
\hline 35-Piperaceae & Piper arboreum Aubl. & Jaborandi \\
\hline 36-Proteaceae & Roupala montana Aubl. & Carne-de-vaca \\
\hline \multirow{4}{*}{ 37-Rubiaceae } & Alibertia sessilis K. Schum. & Marmelada-de-cachorro \\
\hline & Palicourea rigida $\mathrm{H}$. B. K. & Bate-caixa, gritadeira \\
\hline & Rudgea viburnoides (Cham.) Benth. & Erva-de-bugre \\
\hline & Tocoyena formosa (Cham. \& Schltdl.) K. Schum. & \\
\hline 38-Rutaceae & Zanthoxylum rhoifolium Lam. & Mamica-de-porca \\
\hline 39-Sapotaceae & Pouteria ramiflora (Mart.) Radlk. & Pessegueiro-do-cerrado \\
\hline 40-Solanaceae & Solanum lycocarpum A. St.-Hil. & Fruta-de-lobo \\
\hline \multirow{2}{*}{ 41-Styracaceae } & Styrax camporum Pohl & Macieira \\
\hline & Styrax ferrugineum Nees \& Mart. & São-josé \\
\hline 42-Symplocaceae & Symplocos nitens (Pohl) Benth. & \\
\hline 43-Verbenaceae & Aegiphila lhotzkiana Cham. & Assapeixe-branco \\
\hline \multirow{3}{*}{ 44-Vochysiaceae } & Qualea grandiflora Mart. & Pau-terra \\
\hline & Qualea multiflora Mart. & Pau-terrinha \\
\hline & Qualea parviflora Mart. & Pau-terrinha \\
\hline
\end{tabular}

R. Árvore, Viçosa-MG, v.27, n.3, p.413-419, 2003 
Quadro 2 - İndices de diversidade de Shannon (H’) em cerrados da Chapada Pratinha (Felfili et al., 1993) e de Abaeté

Table 2 - Shannon diversity Index (H') in cerrrados in Chapada Pratinha (Felfili et al., 1993) and Abaeté

\begin{tabular}{|c|c|}
\hline \multicolumn{1}{|c|}{ Cerrado } & $\mathrm{H}^{\prime}$ \\
\hline Abaeté-MG & 3,59 \\
\hline Estação Ecológica de Águas Emendadas - DF & 3,62 \\
\hline Patrocínio-MG & 3,53 \\
\hline APA Gama Cabeça-de-Veado-DF & 3,56 \\
\hline Parque Nacional de Brasília-DF & 3,34 \\
\hline Silvânia-GO & 3,31 \\
\hline Paracatu-MG & \\
\hline
\end{tabular}

Quadro 3 - Parâmetros fitossociológicos do cerrado de Abaeté. DA-densidade absoluta, DR-densidade relativa, DoAdominância absoluta, DoR-dominância relativa, FA-freqüência absoluta, FR-freqüência relativa e VI-valor de importância

Table 3 - Phytosociological parameters of cerrado in Abaeté. DA-absolute density; DR-relative density; DoA-absolute dominance; DoR-relative dominance; FA-absolute frequency; FR-relative frequency; VI-importance value

\begin{tabular}{|c|c|c|c|c|c|c|c|}
\hline Espécie & DA & DR & DoA & DoR & FA & FR & VI \\
\hline Xylopia aromatica & 430,0 & 9,63 & 2,7449 & 9,18 & 100,00 & 3,40 & 22,21 \\
\hline Myrcia lingua & 390,0 & 8,74 & 1,8062 & 6,04 & 100,00 & 3,40 & 18,18 \\
\hline Caryocar brasiliense & 140,0 & 3,14 & 3,6693 & 12,27 & 73,33 & 2,49 & 17,91 \\
\hline Eugenia dysenterica & 300,0 & 6,72 & 2,3636 & 7,91 & 86,67 & 2,95 & 17,58 \\
\hline Byrsonima intermedia & 300,0 & 6,72 & 1,0662 & 3,57 & 100,00 & 3,40 & 13,69 \\
\hline Brosimum gaudichaudii & 256,7 & 5,75 & 1,0116 & 3,38 & 80,00 & 2,72 & 11,86 \\
\hline Erythroxylum daphnites & 173,3 & 3,88 & 1,1905 & 3,98 & 80,00 & 2,72 & 10,59 \\
\hline Qualea grandiflora & 136,7 & 3,06 & 1,3254 & 4,43 & 80,00 & 2,72 & 10,22 \\
\hline Byrsonima verbascifolia & 170,0 & 3,81 & 0,8089 & 2,71 & 86,67 & 2,95 & 9,46 \\
\hline Astronium fraxinifolium & 96,7 & 2,17 & 1,3237 & 4,43 & 80,00 & 2,72 & 9,31 \\
\hline Qualea multiflora & 156,7 & 3,51 & 0,6283 & 2,10 & 93,33 & 3,17 & 8,79 \\
\hline Acosmium dasycarpum & 160,0 & 3,58 & 0,5760 & 1,93 & 93,33 & 3,17 & 8,69 \\
\hline Miconia albicans & 123,3 & 2,76 & 0,5536 & 1,85 & 80,00 & 2,72 & 7,34 \\
\hline Bowdichia virgilioides & 83,3 & 1,87 & 0,8652 & 2,89 & 73,33 & 2,49 & 7,26 \\
\hline Bauhinia bongardii & 126,7 & 2,84 & 0,1964 & 0,66 & 80,00 & 2,72 & 6,22 \\
\hline Machaerium opacum & 86,7 & 1,94 & 0,5540 & 1,85 & 66,67 & 2,27 & 6,06 \\
\hline Tapirira guianensis & 83,3 & 1,87 & 0,2861 & 0,96 & 66,67 & 2,27 & 5,09 \\
\hline Tabebuia ochracea & 73,3 & 1,64 & 0,3390 & 1,13 & 60,00 & 2,04 & 4,82 \\
\hline Casearia sylvestris & 83,3 & 1,87 & 0,1915 & 0,64 & 66,67 & 2,27 & 4,78 \\
\hline Styrax ferrugineum & 46,7 & 1,05 & 0,4922 & 1,65 & 53,33 & 1,81 & 4,51 \\
\hline Piptocarpha rotundifolia & 50,0 & 1,12 & 0,5291 & 1,77 & 46,67 & 1,59 & 4,48 \\
\hline Lafoensia pacari & 50,0 & 1,12 & 0,5045 & 1,69 & 46,67 & 1,59 & 4,40 \\
\hline Annona crassiflora & 30,0 & 0,67 & 0,6696 & 2,24 & 40,00 & 1,36 & 4,27 \\
\hline Roupala montana & 50,0 & 1,12 & 0,3180 & 1,06 & 53,33 & 1,81 & 4,00 \\
\hline Erythroxylum suberosum & 43,3 & 0,97 & 0,2704 & 0,90 & 60,00 & 2,04 & 3,92 \\
\hline Siparuna guianensis & 96,7 & 2,17 & 0,1447 & 0,48 & 26,67 & 0,91 & 3,56 \\
\hline
\end{tabular}

Continua...

Continued... 
Quadro 3, cont.

Table 3, cont.

\begin{tabular}{|c|c|c|c|c|c|c|c|}
\hline Espécie & DA & DR & DoA & DoR & FA & FR & VI \\
\hline Curatella americana & 30,0 & 0,67 & 0,4833 & 1,62 & 33,33 & 1,13 & 3,42 \\
\hline Pouteria ramiflora & 20,0 & 0,45 & 0,6622 & 2,22 & 13,33 & 0,45 & 3,12 \\
\hline Rudgea viburnoides & 50,0 & 1,12 & 0,1677 & 0,56 & 40,00 & 1,36 & 3,04 \\
\hline Erythroxylum tortuosum & 43,3 & 0,97 & 0,1899 & 0,64 & 40,00 & 1,36 & 2,97 \\
\hline Didymopanax macrocarpum & 30,0 & 0,67 & 0,2701 & 0,90 & 40,00 & 1,36 & 2,94 \\
\hline Ouratea spectabilis & 30,0 & 0,67 & 0,0723 & 0,24 & 53,33 & 1,81 & 2,73 \\
\hline Sclerolobium paniculatum & 16,7 & 0,37 & 0,4277 & 1,43 & 26,67 & 0,91 & 2,71 \\
\hline Connarus suberosus & 30,0 & 0,67 & 0,1053 & 0,35 & 46,67 & 1,59 & 2,61 \\
\hline Aspidosperma tomentosum & 26,7 & 0,60 & 0,1042 & 0,35 & 46,67 & 1,59 & 2,53 \\
\hline Aegiphila lhotzskiana & 26,7 & 0,60 & 0,2113 & 0,71 & 33,33 & 1,13 & 2,44 \\
\hline 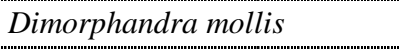 & 23,3 & 0,52 & 0,1834 & 0,61 & 33,33 & 1,13 & 2,27 \\
\hline Styrax camporum & 13,3 & 0,30 & 0,2717 & 0,91 & 26,67 & 0,91 & 2,11 \\
\hline Solanum lycocarpum & 20,0 & 0,45 & 0,0823 & 0,28 & 40,00 & 1,36 & 2,08 \\
\hline Lithraea molleoides & 20,0 & 0,45 & 0,1091 & 0,37 & 26,67 & 0,91 & 1,72 \\
\hline Myrcia tomentosa & 20,0 & 0,45 & 0,1060 & 0,35 & 26,67 & 0,91 & 1,71 \\
\hline Annona coriacea & 20,0 & 0,45 & 0,0947 & 0,32 & 26,67 & 0,91 & 1,67 \\
\hline Stryphnodendron adstringens & 13,3 & 0,30 & 0,1241 & 0,42 & 26,67 & 0,91 & 1,62 \\
\hline Cecropia pachystachya & 10,0 & 0,22 & 0,2715 & 0,91 & 13,33 & 0,45 & 1,59 \\
\hline Tocoyena formosa & 23,3 & 0,52 & 0,0446 & 0,15 & 26,67 & 0,91 & 1,58 \\
\hline Hymenaea stigonocarpa & 6,7 & 0,15 & 0,2832 & 0,95 & 13,33 & 0,45 & 1,55 \\
\hline Annona dioica & 13,3 & 0,29 & 0,0942 & 0,32 & 26,67 & 0,91 & 1,52 \\
\hline Couepia grandiflora & 16,7 & 0,37 & 0,1222 & 0,41 & 20,00 & 0,68 & 1,46 \\
\hline Qualea parviflora & 10,0 & 0,22 & 0,2051 & 0,69 & 13,33 & 0,45 & 1,36 \\
\hline Myrcia rostrata & 13,3 & 0,30 & 0,0385 & 0,13 & 26,67 & 0,91 & 1,33 \\
\hline Byrsonima coccolobifolia & 13,3 & 0,30 & 0,0542 & 0,18 & 20,00 & 0,68 & 1,16 \\
\hline Syagrus flexuosa & 10,0 & 0,22 & 0,0566 & 0,19 & 20,00 & 0,68 & 1,09 \\
\hline Terminalia argentea & 10,0 & 0,22 & 0,1078 & 0,36 & 13,33 & 0,45 & 1,04 \\
\hline Rourea induta & 13,3 & 0,30 & 0,0158 & 0,05 & 20,00 & 0,68 & 1,03 \\
\hline Strychnos pseudoquina & 6,7 & 0,15 & 0,0776 & 0,26 & 13,33 & 0,45 & 0,86 \\
\hline $\begin{array}{l}\text { Indeterminada } \\
\text { ana }\end{array}$ & 6,7 & 0,15 & 0,0563 & 0,19 & 13,33 & 0,45 & 0,79 \\
\hline Davilla rugosa & 10,0 & 0,22 & 0,0179 & 0,06 & 13,33 & 0,45 & 0,74 \\
\hline Banisteriopsis anisandra & 10,0 & 0,22 & 0,0138 & 0,05 & 13,33 & 0,45 & 0,72 \\
\hline Virola sebifera & 10,0 & 0,22 & 0,0082 & 0,03 & 13,33 & 0,45 & 0,71 \\
\hline Alibertia sessilis & 6,7 & 0,15 & 0,0160 & 0,05 & 13,33 & 0,45 & 0,66 \\
\hline Cabralea canjerana & 6,7 & 0,15 & 0,0138 & 0,05 & 13,33 & 0,45 & 0,65 \\
\hline Palicourea rigida & 6,7 & 0,15 & 0,0106 & 0,04 & 13,33 & 0,45 & 0,64 \\
\hline Cybianthus detergens & 6,7 & 0,15 & 0,0073 & 0,02 & 13,33 & 0,45 & 0,63 \\
\hline Annona dioica & 3,3 & 0,07 & 0,0745 & 0,25 & 6,67 & 0,23 & 0,55 \\
\hline
\end{tabular}

Continua...

Continued... 
Quadro 3, cont.

Table 3, cont.

\begin{tabular}{|c|c|c|c|c|c|c|c|}
\hline Espécie & DA & DR & DoA & DoR & FA & FR & VI \\
\hline Tabebuia caraiba & 3,3 & 0,07 & 0,0561 & 0,19 & 6,67 & 0,23 & 0,49 \\
\hline Kielmeyera variabilis & 6,7 & 0,15 & 0,0310 & 0,10 & 6,67 & 0,23 & 0,48 \\
\hline Agonandra brasiliensis & 6,7 & 0,15 & 0,0164 & 0,05 & 6,67 & 0,23 & 0,43 \\
\hline Myracrodruon urundeuva & 3,3 & 0,07 & 0,0344 & 0,12 & 6,67 & 0,23 & 0,42 \\
\hline Piper arboreum & 3,3 & 0,07 & 0,0325 & 0,11 & 6,67 & 0,23 & 0,41 \\
\hline Rapanea umbellata & 3,3 & 0,07 & 0,0179 & 0,06 & 6,67 & 0,23 & 0,36 \\
\hline Cybistax antisyphilitica & 3,3 & 0,07 & 0,0159 & 0,05 & 6,67 & 0,23 & 0,35 \\
\hline Erythroxylum sp. & 3,3 & 0,07 & 0,0106 & 0,04 & 6,67 & 0,23 & 0,34 \\
\hline Hyptis cana & 3,3 & 0,07 & 0,0096 & 0,03 & 6,67 & 0,23 & 0,33 \\
\hline Senna rugosa & 3,3 & 0,07 & 0,0077 & 0,03 & 6,67 & 0,23 & 0,33 \\
\hline Miconia rubiginosa & 3,3 & 0,07 & 0,0068 & 0,02 & 6,67 & 0,23 & 0,32 \\
\hline Enterolobium gummiferum & 3,3 & 0,07 & 0,0068 & 0,02 & 6,67 & 0,23 & 0,32 \\
\hline Vernonia polyanthes & 3,3 & 0,07 & 0,0052 & 0,02 & 6,67 & 0,23 & 0,32 \\
\hline Kielmeyera coriacea. & 3,3 & 0,07 & 0,0048 & 0,02 & 6,67 & 0,23 & 0,32 \\
\hline Zeyheria digitalis & 3,3 & 0,07 & 0,0038 & 0,01 & 6,67 & 0,23 & 0,31 \\
\hline Zanthoxylum rhoifolium & 3,3 & 0,07 & 0,0038 & 0,01 & 6,67 & 0,23 & 0,31 \\
\hline Protium heptaphyllum & 3,3 & 0,07 & 0,0038 & 0,01 & 6,67 & 0,23 & 0,31 \\
\hline Casearia gossypiosperma & 3,3 & 0,07 & 0,0032 & 0,01 & 6,67 & 0,23 & 0,31 \\
\hline Andira fraxinifolia & 3,3 & 0,07 & 0,0032 & 0,01 & 6,67 & 0,23 & 0,31 \\
\hline Symplocos nitens & 3,3 & 0,07 & 0,0032 & 0,01 & 6,67 & 0,23 & 0,31 \\
\hline Diospyros hispida & 3,3 & 0,07 & 0,0026 & 0,01 & 6,67 & 0,23 & 0,31 \\
\hline Bauhinia holophylla & 3,3 & 0,07 & 0,0026 & 0,01 & 6,67 & 0,23 & 0,31 \\
\hline Duguetia furfuracea & 3,3 & 0,07 & 0,0026 & 0,01 & 6,67 & 0,23 & 0,31 \\
\hline
\end{tabular}

A estrutura do cerrado sensu stricto do município de Abaeté mostra um grau de heterogeneidade semelhante à de outros cerrados bem conservados e poderá servir de referência florístico-estrutural para execução de futuras ações de conservação na região.

\section{REFERÊNCIAS BIBLIOGRÁFICAS}

CRONQUIST, A. The evolution and classification of flowering plants. New York: The New York Botanical Garden, 1988. 555 p.

FELFILI, J. M. et al. Análise comparativa da florística e fitossociologia da vegetação arbórea do Cerrado sensu stricto na Chapada Pratinha, DF - Brasil. Acta Botanica Brasilica, v. 6, n. 2, p. 27-46, 1993.
FUNDAÇÃO BIOVERSITAS. Biodiversidade em Minas Gerais: um Atlas para sua conservação. Belo Horizonte: 1998. p. 67-68.

MEIRA NETO, J. A. A. Composição florística e fitossociológica da vegetação de Cerrado Sensu Lato da Estação Ecológica de Santa Bárbara (E.E.S.B.), Município de Águas de Santa Bárbara. Estado de São Paulo. 1991. 98 f. Dissertação (Mestrado em Biologia) Universidade de Campinas, Campinas, 1991.

MUELLER-DOMBOIS, D.; ELLENBERG. H. Aims and methods of vegetation ecology. New York: Wiley, 1974. $547 \mathrm{p}$.

PIELOU, E. C. Ecological diversity. New York: Wiley, 1975. $165 \mathrm{p}$.

SHEPHERD, G. J. Fitopac 1. Manual do usuário. Campinas: UNICAMP, 2001. 93 p.

R. Árvore, Viçosa-MG, v.27, n.3, p.413-419, 2003 